\title{
The conflict of living matter with the mineral world: The pneumoconioses'
}

\author{
A. POLICARD
}

It is a great honour to me to have been invited to give the 'Kettle Lecture' of this year. The memory of the remarkable man of science who was Kettle must inspire me to-day. He was, as you all know, a first-rate experimenter. At the same time, he had a profound knowledge of human disease, especially the infectious diseases. Because he knew microbial pathology so well he had quickly understood the importance of infection in the development of the pneumoconioses, especially of silicosis. From this point of view, the influence of Kettle was very great in the development of our ideas on dust diseases of the lung. More and more, workers in this field are confirming the greater part of these theories.

Kettle was among the first to realize the different actions of different kinds of mineral dust. If he divided them into 'inert' and 'active' dusts, he recognized for all a secondary reaction favourable to the development of bacteria. In this Kettle was a pioneer. Therefore the remembrance of his name and work is not out of place at the beginning of this lecture devoted exclusively to the cellular mechanism of the action of various mineral dusts, that is to say, to the "conflict between living matter and the mineral world', which occurs in the genesis of the pneumoconioses.

I intend to show you some results of research which we, engineers and doctors, J. Charbonnier, A. Collet, J. C. Martin, H. Daniel-Moussard, and myself, at the Centre d'Etudes et Recherches des Charbonnages de France, le CERCHAR, are carrying out on the initial stages only of the pneumoconioses.

We took as our starting point that the mode of origin of the first fibrous reactions of the pneumoconioses is inflammation. Whatever the cause, this inflammation is characterized by the succession of the following well-established processes: vasodilatation of the blood capillaries with an increase in the permeability of their walls, oedema, and diapedesis of the leucocytes, first of polynuclears and then of mononuclears. The inflammation causes a granuloma which becomes fibrous. These processes are always the same qualitatively, but they can vary quantita-

${ }^{1}$ The Kettle Memorial Lecture delivered at Hammersmith Hospital, London, on 1 December 1961. tively according to the nature of the aggressor anfy according to its amount. The attack of mineral duste. is a special aspect of this process, and the problem to determine by which mechanisms mineral dusts entering the tissues provoke inflammation, the origit of fibrosis. In other words, what is the mechanisnff of attack in the cells of mineral dusts?

One point must be made. Mineral particles do not. cause fibrosis by direct action. Many facts demones strate this. If dusts are put in a diffusion chamber 9 a fine porosity which the cells cannot penetrate, n $\vec{P}$ fibrosis is seen in the immediately adjacent tissues This fact is especially demonstrated by the researci done in Great Britain. The dissolution products $0 \vec{b}$ mineral particles do not directly cause fibrosis. \& the other hand, the highly fibrogenic dusts, suckas that of quartz, can be mixed in the middle of a culture in vitro. The culture is not modified if the cells have not absorbed the mineral particles bo phagocyte action, as is the case with cells not capable of phagocytosis such as the myoblasts of the heart of chicken embryo. To provoke fibrosiso mineral dusts have been absorbed by phagocytosis into the cells and have provoked their degeneration thus leading to the production of certain cytoplasmic toxic waste, which lead to an inflammatory reactio more or less active, causing fibrosis. The fibrogenio reaction by mineral dusts is, therefore, an indirect. reaction, implying three essential phases: (1) Penetros tion of the cell by the particles (phagocytosis) (2) degeneration and death of the cell; (3) chemicas dislocation of the cytoplasm (proteolysis) and liberation of protein toxic wastes causing the inflammation and, secondarily, fibrosis. In this sequence of processes, the cell acts as a microbe

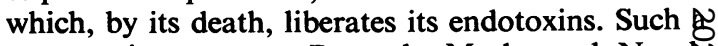
concept is not new. Recently Marks and Nagew schmidt have also advanced it. The lesion of the phagocytic cells, and not a direct action of the dus is the origin of the fibrosis.

The first of these processes, phagocytosis, is fap from being simple. It sets in motion physicochemicat processes and biological complexes as yet ill-define $\Phi_{-}$ It will not be dealt with here. The third, the liberatiog of toxic inflammatory products, has been studied fort 
many years. It is not necessary to quote here the well-known work of Menkin, of Spector, and of many others. Our research has aimed at identifying the chemical nature of the toxic wastes. They seem to be peptides of low molecular weight which provoke the vasodilatation of the capillaries and an increase in their permeability. These problems are very difficult and have mostly been studied on the more usual types of inflammation but not on that provoked by mineral dusts. Many biochemical studies remain to be done. The process will not, therefore, be dealt with here. All that will be dealt with in this lecture are the changes produced in cells which have phagocytosed different kinds of mineral particles. These problems are far from being simple. Therefore, they have to be well defined.

Indeed, the different kinds of mineral dusts act in very different ways towards the cells which have phagocytosed them. Let us consider, for example, a macrophage (histiocyte) of lung tissue. Its morphological modifications, its biological behaviour, its resistance are quite different, depending on which particles are involved: quartz, amorphous silica, coal, iron oxide, lime, mica, etc. When we say that a substance is very fibrogenic, like quartz, we mean that its dust, after phagocytosis, provokes a rapid and severe degeneration in the cell, with the liberation of particularly toxic protein products which start a strong inflammatory reaction followed by an accentuated fibrosis. With a substance called 'inert', coal, for example, the dust provokes few or no cellular alterations, causes no degeneration, no toxic products, and therefore neither inflammation nor, consequently, fibrosis. On this basis, at CERCHAR we have been led to undertake the systematic study of a certain number of dusts met with in the mineral industry from the point of view of their action on the phagocytic cells and on their capacity to bring about the production of toxic products which generate fibrosis.

For this research on 'pathological minerology' we have till now used two experimental methods. One, essentially dynamic, consists of studying the effect of different dusts on living phagocytic macrophages (histiocytes) by phase-contrast microfilm examination. The other, entirely static, method relies on the use of an electron microscope: with this it is possible to obtain information on the effect of dusts on the ultramicroscopic structures of the cells. As can be seen, these two methods are complementary.

\section{EXAMINATION OF THE MACROPHAGES} IN VIVO

The technique used is well known. Into the peritoneal cavity of adult rats is injected a small quantity of a suspension, in physiological solution, of exactly calibrated particles below $3 \mu$. After 12 hours, the peritoneal exudate is drawn into a pipette. One drop is deposited on a slide, covered with a cover-slide, sealed with paraffin, placed under the phase-contrast microscope and observed at $37^{\circ}$. The cells which have not been centrifuged or washed are examined cinematographically, pictures being taken every second. They are projected at a minimum rate of 20 to 30 a second. This method of study is very valuable in assessing the multiple aspects of the behaviour of the macrophages and of their varying degeneration.

In the exudate, beside the very mobile polynuclear cells which have usually not phagocytosed any dust, there are macrophages more or less loaded with particles. Some are dead and full of dust; others are very active. Between these two extremes there is a series of intermediary stages. As you will see later, it is easy to judge the state and the behaviour of these cells.

Taken as a whole, and not in detail, it is possible to observe the following results: There are nearly always histiocytes which phagocytose the dusts. The dusts are rarely found in polynuclear cells. The changes in the histiocytes (macrophages) vary greatly according to the nature of the mineral. It is possible to distinguish three kinds of behaviour:

1 In the 'inert' kind, seen with coal, haematite, and oxide of titanium, for example, the particles present do not appreciably modify the behaviour of the phagocyte as it continues to move. However, if the particles are excessively numerous, movement is slowed down. When the cells die in the normal way, they appear as rigid sacs, displaced in their entirety.

2 With quartz, the presence of a small number of particles suffices to immobilize the macrophage. Their overall activity diminishes. The internal movements of the cytoplasm are slowed down or suppressed. Certain cells show an increased bubbling with these particles and the exudate frequently contains cellular debris or collections of particles, the remains of dead and dislocated cells.

3 A type that occurs frequently is the vacuolar. The mineral dust causes the appearance of large vacuoles which can reach a size of 3 to $4 \mu$, with clear contents and definite limits, but without membrane. With calcite, some mineral particles are enclosed in the vacuole and show an intense Brownian action. These are the particles in the process of dissolution. The remainder of the cytoplasm appears to be degenerate and dense. With other minerals, the vacuoles are similarly arranged but the particles are not usually lodged in the vacuole; they are next to it in the cytoplasm.

You will be able to observe the different aspects 

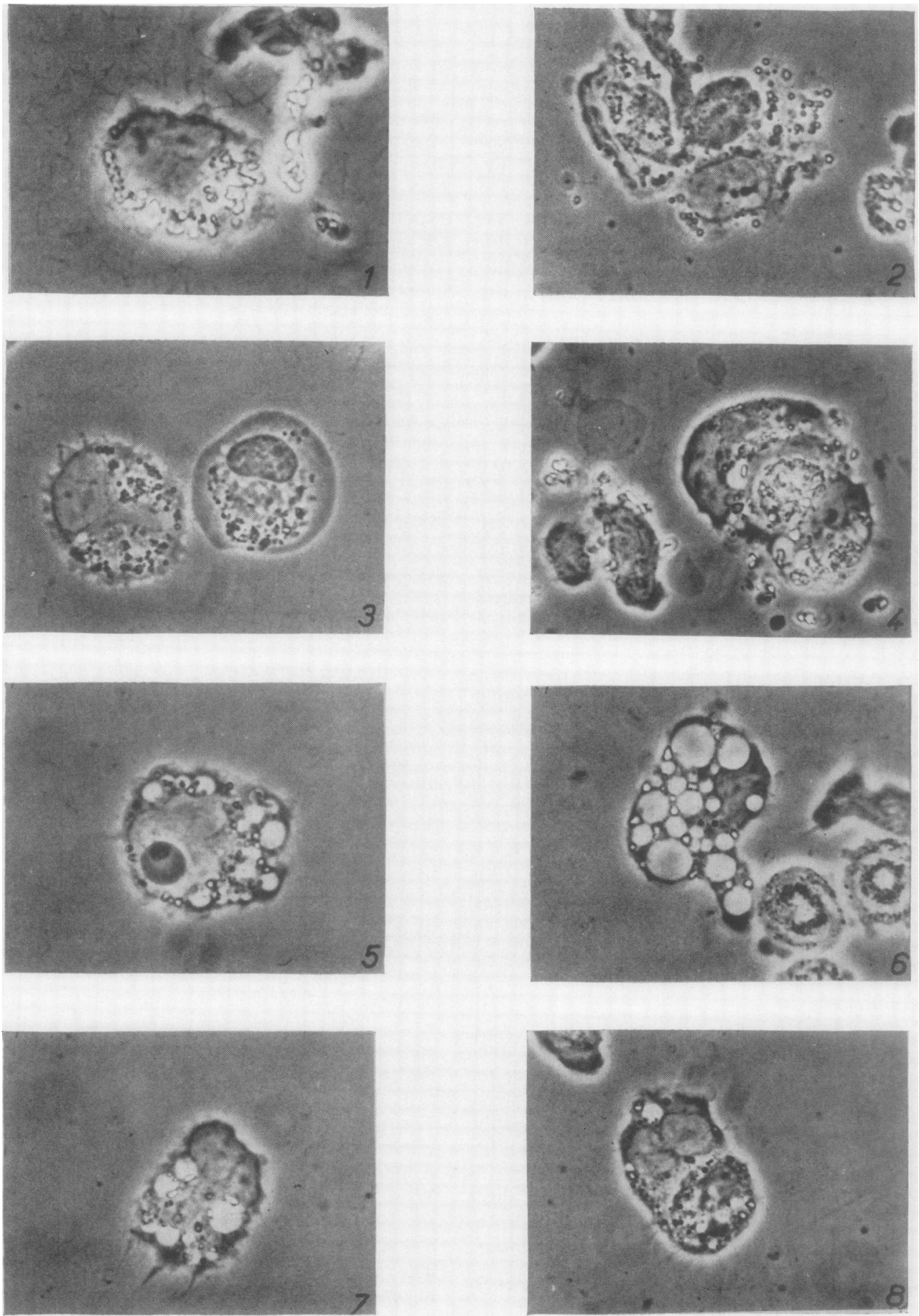

FIG. 1. Microcinematographic films with phase contrast of peritoneal macrophages phagocytosing various mineral dusts. 1 , quartz; 2 , cristobalite; 3 , tridymite; 4 , kaolin; 5 and 6 , silica-gel; 7 , mica; 8 , montmorillonite. 
shown by dust macrophages and their variable degeneration. It is, nevertheless, necessary to note the yet incomplete character of these results from the point of view of morphological details. Phasecontrast, very useful for following the living elements in their general behaviour, is not very helpful from the structural point of view.

\section{FINDINGS WITH THE ELECTRON MICROSCOPE}

We have attempted to complement the results obtained by phase-contrast microcinematography by showing with the electron microscope the modifications brought about in the protoplasmic ultrastructures by the action of phagocytosed mineral dusts. Naturally, this technique is static, but it complements the examination of the living elements.

We examined the pulmonary tissue of rats after intratracheal injection of dust suspensions and also the nodules of dust formed in the omentum after injection of dust into the peritoneal cavity. These various examinations were made six days after the injection of the dust. Fixation, cutting of sections, and examination by the electron microscope $\mathbf{R ~ C ~ A ~}$ were similar. The sections were made with the Fernandez Moran diamond knife, using a very hard medium. Apart from quartz, all the other substances examined could be cut in ultrafine sections of 300 to 400 angstroms.

1 INERT MINERAL SUBSTANCES Certain minerals seem to have no appreciably injurious action on protoplasmic structures. Such are coal dust, haematite dust, and titanium oxide dust. On contact with the particles, cytoplasm does not show any visible modification even at high-power resolution. Neither the nucleus, nor the mitochondria, nor the endoplasmic reticulum show any changes. There are no vacuoles visible around the particle. Nuclei 200 or 300 angstroms from the particles show no modification. Recently we have observed the equally inoffensive character of diatomite (Kieselguhr), a fossil silica of biological origin. These facts confirm in particular the theory of the innocuous nature of coal towards cellular protoplasm. The results are worth emphasizing from the practical point of view.

2 MINERAL SUBSTANCES COAGULATING PROTOPLASM Certain minerals, such as certain varieties of silica, have a distinctly coagulating action on the cytoplasm. If the action of quartz is almost impossible to study with the electron microscope because it is too hard, one can obtain ultra-thin sections of cells enclosing particles of condensed silica, amorphous silica, less hard but nevertheless toxic. Arcund these particles of silica, or around their mass, can be seen homogeneous and dense clouds of cytoplasm which seem to have resulted from the denaturation of the cytoplasm by coagulation. Occasionally in the centre of these clouds of cytoplasmic coagulation can be seen clear zones of irregular vacuolation. Their significance is not yet specified.

Between the coagulated zones and the remainder of the cytoplasm the limit is undefined and it is never marked by a membrane. On the other hand, a membrane does appear in cases of secondary phagocytosis of fragments of dead cells containing silica. For this reason, these pictures of secondary phagocytosis are easy to distinguish from primary phagocytosis of silica particles.

The problem of the coagulating ability of silica on protein is still unsolved. Following the recent work done in England and Germany on the coagulating ability of silicic acid, more or less polymerised, on different proteins, it seems that albumin is not precipitated; that the gamma-globulin and fibrinogens, which are very unstable, precipitate easily, and that the alpha and beta-globulins, which are less unstable, precipitate less easily. However, any correlation between the cytological observations and biochemical findings does not yet seem possible.

Generally the densification of the cytoplasm around isolated particles is weak and sometimes barely perceptible. On the other hand, in the more usual instances of accumulated silica particles, the cytoplasmic densification and vacuolar spaces are always well defined. This may be due to the larger area of contact offered in the case of the accumulations.

3 MINERAL SUBSTANCES PROVOKING THE FORMATION OF VACUOLES Examination by phase-contrast microcinematography shows the existence of a specific vacuolar reaction of the cells vis-à-vis certain minerals. Study with the electron microscope complements these observations.

The formation of vacuoles under the influence of mineral particles is not a simple process; they present varied morphological types. With particles of calcium carbonate (calcite) the vacuoles formed are large (up to $3 \mu$ ). They are round, with distinct limits, but without membranes. Their content is slightly dense, without having, however, the empty transparency of the spaces left by the technical eradication of the particles. In these vacuoles free particles are found in ccurse of dissolution and, as can be seen in vivo, they show very active Brownian movement. The cytoplasm outside the vacuoles appears dense, either in the whole extent of the cellular body, or at the level of certain foci, often extensively. This densification, weak but well 


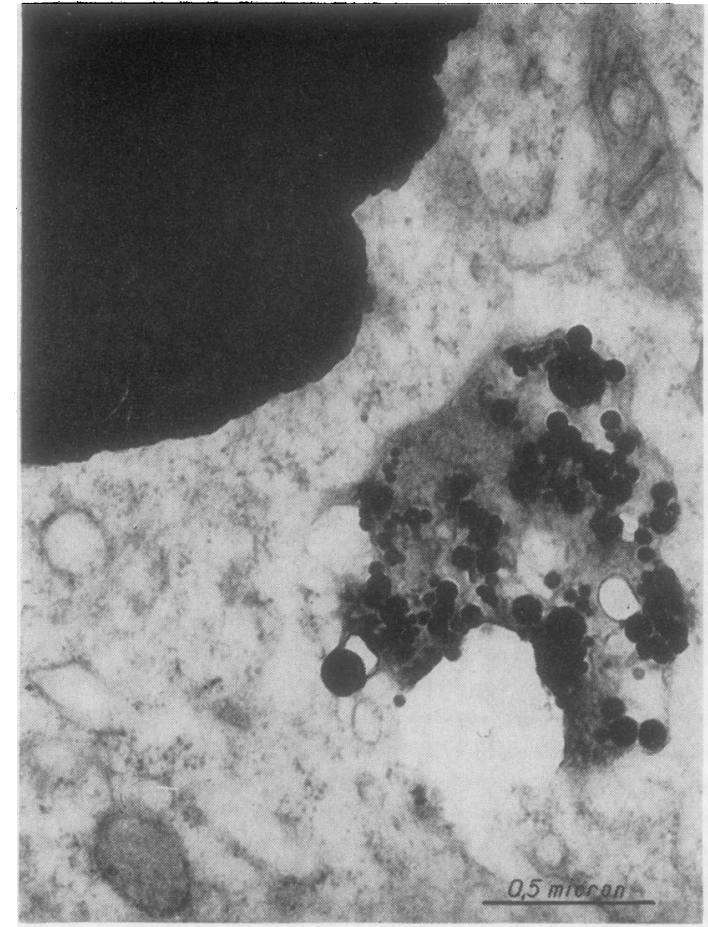

FIG. 2

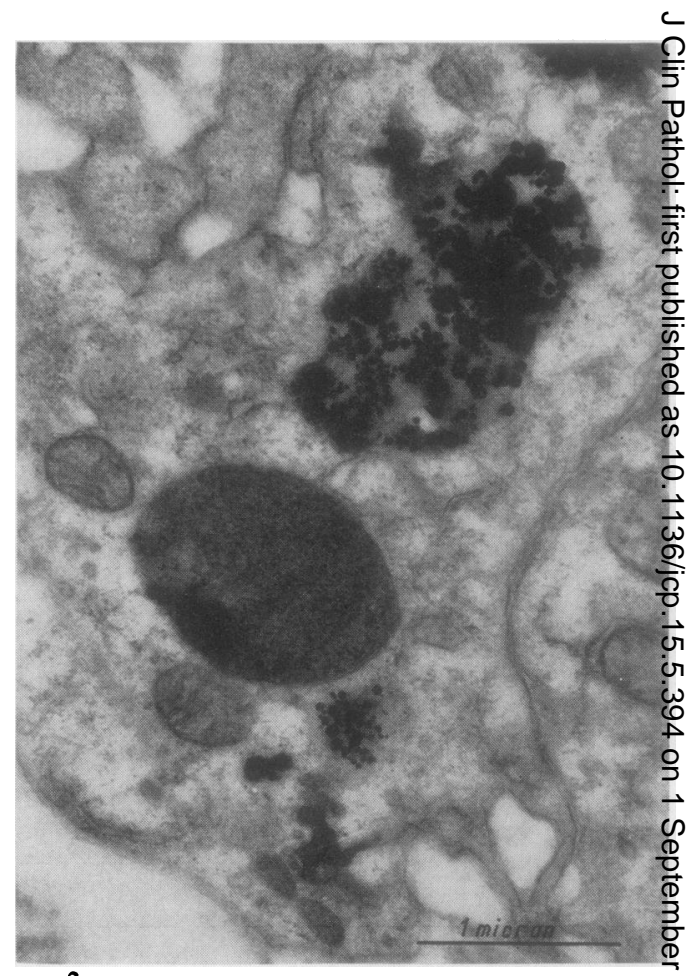

FIG. 3

FIG. 2. Electronmicrograph of a macrophage with coal-particle (top) and an aggregate of amorphous silica particie (Fransil), surrounded by a cloud of denatured cytoplasm.

FIG. 3. Electronmicrograph of an aggregate of amorphous silica particles (Fransil) surrounded by a cloud of denat cytoplasm.

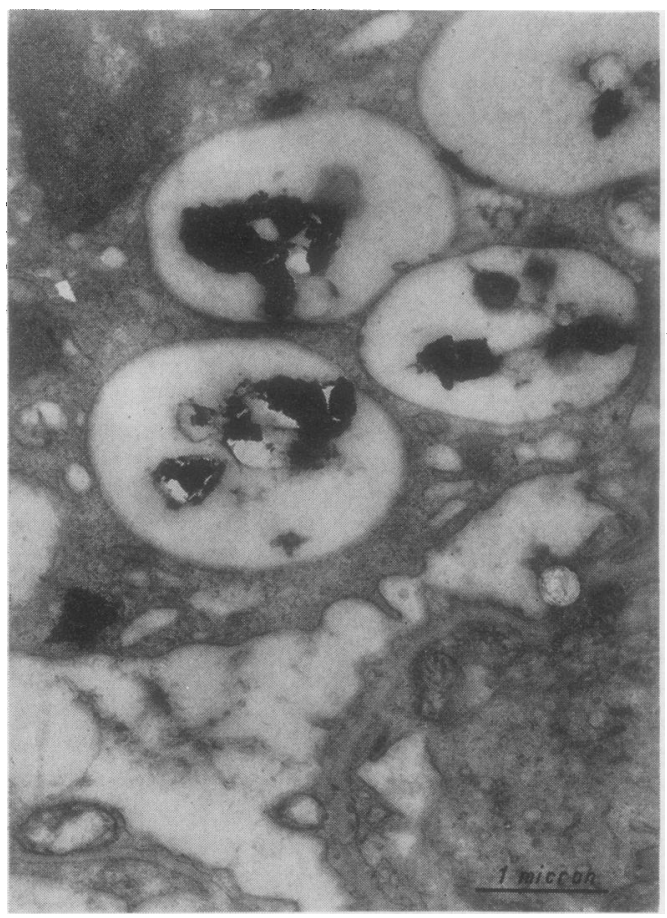

FIG. 4

FIG. 4. Electronmicrograph of a macrophage with calcite particles in light vacuoles.

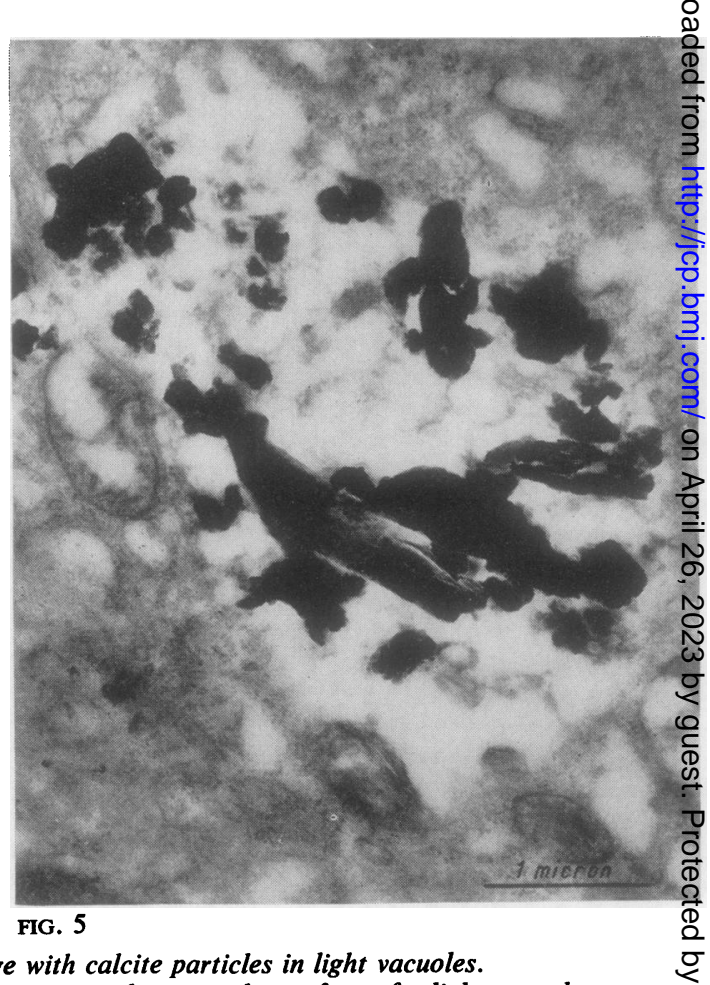

FIG. 5. Electroninicrograph of a macrophage with mica particles upon the surface of a light vacuole. 
defined, is accompanied by ultrastructural modifications exceedingly difficult to analyse.

With particles of mica, there is also vacuolization of the cytoplasm. Unlike those of calcium, mica particles are not generally found inside the vacuole, but are definitely within the cytoplasm in contact with the vacuoles to which they seem attached. In some instances, the vacuole is distinct and quite spherical. In other instances, more numerous, the vacuolar disposition is indefinite and instead there is vacuolization of a cellular region which encloses the particles. These show no sign of dissolution. On the other hand, the cell shows no signs of alteration with densification. On contact with the dust, the ultrastructure of the cytoplasm does not appear to be appreciably modified although this appearance has not been verified. Anyhow, there exist incontestable differences between the vacuolization by calcite and that by mica.

\section{CONCLUSIONS}

The following conclusions can be reasonably drawn from the results shown.

The first point to be made is that the results observed do not lead to any conclusion on the fundamental problem, so much discussed in recent years, of the mechanism of the harmful action of certain mineral particles. However, the problem should be transferred from the level of the tissue to that of the cell. But the problem does not change. Is the action of a quartz particle due to the fact that it throws off around itself a toxic substance, silica? Or, because at its surface there are adsorbed and modified cytoplasmic elements-structural proteins or enzymes-indispensable to cell life? Our observations do not give us the answer. Nevertheless, it should be remarked that coal, with an adsorbent ability which is occasionally very high, is not noxious to cytoplasm.

A second conclusion can be drawn from our observations. Cytoplasm can apparently be altered by dust in two ways: by coagulation (that is to say by irreversible denaturation), or by vacuolization, and by proteolysis to more simple protein complexes. This is well known in pathological cytology. The post-mortem degeneration of cells is produced in two principal ways: coagulation necrosis implying suppression of all enzymes, and autolytic proteolysis involving dislocation of the cytoplasm proteins. The coagulated cell can, however, be disrupted, but more slowly and by proteases of external origin (heterolysis). In autolytic proteolysis, there is a great deal of liberated waste, polypeptides in particular. These find themselves in a favourable medium and set off the inflammatory reaction early. It should be possible to apply these well-known findings of general pathology to the action of mineral dusts. However, to show the truth of this conception, it would be necessary to isolate the cellular wastes and to study experimentally their capacity to provoke inflammation and fibrosis. It is to be hoped that biochemical and toxicological research may be carried out on these fundamental points.

From our work other conclusions can be drawn. First, it is necessary to define the particular cellular effects of the different mineral dusts. We have been able to distinguish, at least provisionally, three principal types, and these types should be worth investigating further. It is possible that other types of mineral injury to the cells will be found and should be sought. In fact, a new chapter of cytopathology is being opened, a kind of 'mineral cytopathology' in which it is necessary not only to define morphologically and physiologically the action of each kind of mineral, but also to understand the mechanism. This project is certainly a very fomidable one, but it is necessary and will be fruitful. Moreover, yet another chapter should be opened in mineralogical cytopathology, that of the effect of a mixture of mineral dusts. A cell can phagocytose particles of several types of minerals. What are then the reactions of its cytoplasm? What common influences would the dusts exert over one another? Since it frequently occurs in industry that the inhaled dusts are mixed, the problem is therefore of great practical interest. In the course of research, which is still only beginning, we have already been able to observe that particles of diatomite (Kielselguhr), by themselves innocuous and non-fibrogenic when added to quartz particles, reduce the fibrogenicity of the latter. How can this be explained? No one as yet has propounded a theory. However, one can only think that at the beginning of the phenomenon there is perhaps a modification of the capacity of quartz to alter the cytoplasm when the latter would not suffer proteolysis as before and so would not produce the toxic proteins which cause inflammation. We are now pursuing our researches on these points.

I have spoken of the difficult and still uncertain problems of pathological cytology. The biological field which we have surveyed is full of pitfalls and stumbling blocks, and in this cytopathological and cytomineralogical jungle progress is difficult.

\section{COMMENT BY PROFESSOR J. H. DIBLE}

We have listened to a most fascinating and erudite lecture from the doyen of French pathologists. He early used a striking phrase that set the whole tone of his discourse: 'The conflict between living matter and the 
mineral world', and with this as his text has dealt with the subject not from the narrow speciality of silicosis but from the wider and more far-reaching viewpoint of general pathology, on so many aspects of which his work has touched.

The interaction of living and dead matter within the body is an old and fascinating problem in general pathology that has engaged the interest of pathologists and clinicians from the earliest times; beginning, I suppose, with the deposition of calcium salts in the formation of bone, progressing from this to the reaction of the tissues to dead bone, and to the causes and effects of deposited bone salts, and even of bone itself, in aberrant situations, such as the degenerating arterial wall, old uterine myomata and the like. In the beginning of the century the effects of metal within the tissues aroused the interest of surgeons in such matters as the implantation of wire mesh for the repair of herniae, of metal splints in the treatment of fractures, and of gold leaf to prevent adhesions between the cerebral membranes, and still more recently of surgical prostheses in joints $\stackrel{0}{\overrightarrow{5}}$ Finally, we come to that group of diseases of grea? social importance-the pneumonoconioses. It was these that especially interested Edgar Hartley Kettle and he made some important contributions to their study. Bug probably his greatest was the arousing of interest ito their investigation, and surely it is of significance tha two of the schools in which he held the chair of patho@ logy have become foci of the most active work in this field: the school of lung pathology developed underp Gough at Cardiff, and that inspired and directed here a Hammersmith by King.

You, Professor Policard, used another striking phrase when you referred to the beginning of 'a new chapter og cytopathology'. In the address you have given us yog have indicated its lines of development by the employ=ment of the most modern techniques. We have been privileged to hear from you this afternoon the intro duction, if not the contents and first chapter, of this booke which the future will write. 